Wenn unsere Literatur für diese in den Entwickelungsvorgängen gegebenen Vaginalatresien kaum irgend ein reines Beispiel aufweist, so liegt dies darin, dass derartige Fälle selten zur Section kommen, und wenn dies geschieht, die Form und Lage der in Betracht kommenden Stelle meistens dureh Druek des Menstrualblutes oder durch Cohabitationsversuche verändert ist, Die Durchsicht der von Kus smaul ${ }^{1}$ ) und Fürst ${ }^{2}$ mit so grossem Fleisse gesammelten Beobachtungen giebt zahlreiche Belege hierfür.

\title{
Ein Beitrag zur Lehre vom Vaginismus.
}

\author{
Von \\ Heinrich Fritsch, \\ Privatdocent in Halle.
}

Noch heute stehen sich die Ansichten iiber die Aetiologie, Wesen und Behandlung des Vaginismus schroff gegeniiber. Während die Einen, den entzündlichen Charakter betonend, mit einer antiphlogistischen Therapie stets Heilung erzielen wollen, werden von Auderen grosse, blutige Operationen für nöthig befunden. $I_{n}$ der neuesten Zeit hielt man den Vaginismus fïr etwas der Fissura ani durchaus Analoges, und wollte ihn durch gewaltsame Dilatation heilen.

Ohne auf die verschiedenen Ansichten einzugehen, ist zunächst zu bemerken, dass die Diagnose auf Vaginismus verschieden gestelit wird. Dies beweisen schon die verschiedenen Angaben über die Häufigkeit. Gehören alle von Sims geschilderten, schweren Symptome zum Bilde des Vaginismus, so ist er gewiss eine sehr seltene, durch Antiphlogose nicht zu beseitigende $\triangle$ ffection. Wird dagegen jede stärkere Hyperästhesie, welche als Folge einer traumatischen Vulvitis sich häufig findet, für Vaginismus erklärt, so sind diese Fälle häufig, und ihre Heilung durch Antiphlogose und die Vermeidung des Trauma leicht zu erzielen. Bei Stellung der Diagnose aber ist gewiss die Hyperästhesie als das Wichtigste zu betrachten, denn die Reflexkrämpfe können sich in ausgebildeter Weise nur bei Nulliparis finden.

Der mitzutheilende Fall schien mir besonders lehrreich zu sein.

1) Mangel, Verkümmerung und Verdoppelung der Gebärmutter. Würz: burg 1859 .

2) Monatsschrift für Geburtskunde, Bd. XXX, 1867. Hft. 2 u. 3. 
Frau A., 38 Jahue alt, Yutter zweier Kinder von 15 and 6 Jahren, wurde Anfang des Jabres 1876 zum dritten Male schwanger und abortirte am 10. Mai. Acht Tage nach dem Abort trat eine heftige Blutung ein. Niemals hatte Patientin vorher oder nachher Fluor albus, Seit dem Abort ist Patientin abgemagert und leidet an fortwährenden, quidenden Schmerzen. Sie wurde schon von einem anderen Arzte mit Vaginaldouchen, und als diese $z u$ schmerzhaft waren, mit Sitzbädern behandelt. Ein Erfolg dieser Therapie trat nicht in die Erscheinung.

Patientin giebt an, dass ihre ganzen "Schamtheile" geschwollen seien. Sie fühle deutlich, dass sich etwas ,herausdränge", das Gehen sei unmöglich, sie müsse fast stetis liegen. Das „Brennen" und die bei jeder Bewegung zunehmenden Schmerzen raubten jeden Schlaf und machten jede häusliche Beschäftigung unmöglich. Patientin wünscht sehnlichst von ihren Leiden befreit zu werden.

Der Ehemann sagt aus, dass seit derZeit, in welcher die Schmerzen auftraten, seine Frau immer melancholischer werde. Sie lache nie, weine Nächte lang und esse wenig. Der Coitus sei wegen übergrosser Schmerzhaftigkeit nicht mehr ansgeübt. Er selbst glaube an beginnende geistige Störung. Schon oft habe man eine genaue Beobachtung wegen eventueller Selbstmordversuche für nöthig gehalten. Auch Patientin erzählt von freien Stuicken, dass sie sich mit diesem Gedanken getragen habe.

Bei der Inspection der Vulva zeigen sich die äusseren Genitalien frei von jeder Entzündung. Sobald die Sehamlippen auseinander geklappt werden sollen, entstehen erhebliche Schmerzen. Pat. klammert beide Beine zusammen, und geräth in die höchste Aufregung. Sie weint und sagt, es wären dies die nämlichen, nur verstärkten Schmerzen, wie sie dieselben immer habe. Bei jeder Berührung der Vulva, auch nur durch Kleidungsstücke steigere sich die Schmerzhaftigkeit, dagegen nähme sie bei geistigen Aufregungen nicht zu. Auf Zureden gelingt die Exploration, welche merkwiirdigerweise keinerlei Schmerzen macht. Weder eine Gesehwulst, noch Secrete, noch irgend etwas Pathologisches ist zu entdecken.

Als ich der Patientin erklärte, dass weder ein Vorfall noch irgend eine Geschwulst oder Schwellung vorhanden sei, gerieth sie ausser sich, und bat dringend, sie in Behandlung zu nehmen. Es wurde auf die Vulva Tannin gepulvert, Sitz- und Vollbäder verordnet, Bleiwasserumschläge applicirt, und die ganze Affection für eine reine Neuropathie gehalten. Patientin glaubte eine geringe Besserung zu verspüren. Zufällig wurden bei einer Bepuderng mit Tannin die kleinen Sehamlippen auscinandergezogen. Da, als der Pinsel gerade in die Mitte unter der. Clitoris gerieth, fing Patientin plötzlich laut an zu schreien, es entstand ein wahrer Schmerzparoxysmus. Hier also fand sich eine beschränkte Stelle, wo das Centrum der Schmerzempfindung existirte, Line genauere Untersuchung war schon wegen der enormen Angst der Patientin numöglich. Es wurde nareotisirt und besehlossen, in der Narcose die betreffende Stelle za ätzen oder 
zu excidiren. Auch in der Narcose war die Hyperästhesie auffallend. Als schon der ganze Körper aufgehört hatte zu reagiren, warf sich Patientin sofort herum, wenn die kleinen Schamlippen auseinandergezogen wurden. Erst nach Einleitung einer sehr tiefen Narcose gelang es, das Feld gut sichtbar zu machen. Es zeigte sich gerade in der Mitte, 3/4 Cm. nach abwärts von der Clitoris eine kleine $5 \mathrm{Mm}$. lange, einen Millimeter breite Fissur. Sie sah gelblich aus, ibre Umgebung, aber nur in ganz beschränkter Ausdehnung, war hochroth gefärbt. Die Stelle schien zu klein, um eine Excision nöthig zu machen, deshalb ätzte ich sehr ausgiebig mit Argentum nitricum. Danach applicirte ich Eis, welches erst nach vier Tagen entbehrt werden konnte. Binnen kurzem stiess sich der Schorf ab, es entstand eine granulirende, bei der Beriuhrung nicht schmerzhafte Ober-

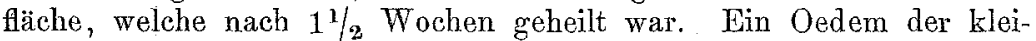
nen Schamlippen wurde durch Bepinselung mit Acidum pyrolignosum zum Schwinden gebracht. Dann blieb die Heilung vollständig.

Wir sehen also, dass der gesammte Symptomencomplex des Vaginismus sich findet, ohne dass die. Vagina oder die Vulva entzündlich erkrankt ist; eine gewaltsame Dilatation, eine Abtragung der Hymenreste wäre ebensowenig wie eine blose Antiphlogose von Erfolg begleitet gewesen. Erst als die kleine Rhagade entdeckt wurde, konnte zweckentsprechend eingegriffen werden.

Interessant ist in unserem Falle die Täuschung der Patientin über den Grund und den Sitz des Leidens: die perverse Sensation. Patientin war fest überzengt, an "Vorfall" und "Schwellung" zu leiden. Pathologische und instinctive Empfindungen werden ja namentlich von Hysterischen in der eigenthümlichsten Weise gedentet.

So behandelte ich eine Hysterica, welche so schlaffe Bauchdecken hatte, dass man die inneren, weiblichen Genitalien auf das vorzüglichste abtasten konnte. Beim Liegen bewegten sich mit dem Pulse der Aorta die Bauchdecken. Patientin fuillte diese Pulsationen und behauptete, in Folge des Genusses von Flusswasser einen Fisch im Leibe zu haben. Die Grösse und Lage des Fisches wurde so genau beschrieben, dass die abnorme Sensation deutlich auf beide Nierenarterien zu beziehen war.

Noch charakteristischer ist der folgende Fall. Eine alte, anämische, decrepide Puella publica glaubte einen grossen Vorfall zu haben. Sie beschrieb deutlieh, dass beim Uriniren ein grosser Körper die Vulva auseinanderpresse und hervorträte, dann zöge er sich unter starken Schmerzen wieder zurück. Als ich bei der Untersuchung die Existenz cines Vorfalles leugnete, war es der Patientin ganz unbegreiflich. Erst bei einer späteren Untersuchung entstanden beim Catheterisiren die furchtbarsten Schmerzen. Patientin schrie laut auf und unter spasmodischer Action der Blase trat eine erbsengrosse, gestielte, hochrothe Harnröhrencarunkel in die Urethralöfnung. Als ich die Geschwulst mit der Pincette anfassen wollte, um sie behufs der Abtragung herabzuziehen, waren die Sehmerzen so enorm, dass ich zu narcotisiren gezwungen war. Hieranf liess sich die Carunkel, 
deren Stiel über einen Centineter lang war, leicht hervorziehen und abschneiden. Die Heilung war vollständig.

Dergleichen Fälle erinnern sehr an die von $\mathrm{Köppel)} \mathrm{beschrie-}$ benen reflectirten Psychosen. In unserem ersten Falle hatte das viermonatliche, geringe locale Leiden Patientin körperlich und geistig so heruntergebracht, dass man eine exquisite Melancholica zu sehen glaubte. Leicht ist es denkbar, dass eine wirkliche Psychose entstanden wäre, wenn man die Rhagade nicht bald zur Heilung gebracht hätte. Ist doch iiberhaupt die Hysterie eher eine reflectirte Psychose als eine Reflexneurose zu pennen!

Zum. Schluss noch ein Wort über den Ausgangspunkt der Krank heit. Er liess sich in unserem Falle, wenn auch unter Schwierigkeiten, auffinden, gewiss wäre von Vielen dieser Fall, wie es von mir zuerst gesehah, als reine Neuropathie gedentet. Deshalb kann man der Vorschrift, bei Vaginismus die Vulva und den.Introitus a ufs genaueste uach kleinen Wunden zu untersuchen, nur zustimmen. Entweder mass eine Entzïndung oder eine Fissur bestehen.

Dass übrigens eine Verwechselung mit dep pathogenetisch so ähulichen Analfissur stattfinden kann, lehrte mich folgender Fall.

Eine Frau war vor einem Jahre zum ersten Male entbunden. Das Kind war in Steissla ge geboren. Der Arzt hatte den Kopf ungeschickt entwickelt und den Damm usque in anum gerissen. Die Ruptux war nicht genäht. Ein eigentlicher Sphincter externus fehlte, wenn anch eine Scheidewand bis nach unten geblieben war. Patientin litt von der Entbindung her an übermässig hartem Stuhlgang und enormen Schmerzen bei demselben. Als wiehtigstes Leiden aber, wegen dessen Patientin ärztliche Hülfe suchte, galt ihr die Unmöglichkeit der Cohabitation. Sobald dieselbe ausgeübt werden sollte, traten Drang nach unten, Blasenkrampf und erhebliche Schmerzen ein, als "wenn alles zerrisse". Bei der genauen Untersuchung fanden sich an der Analseite der dünnen Rectovaginalwand sieben verschieden lange Fissuren. Eine Behandlung in der gewöhnlichen Weise, eine Excision, tiefe Incision oder gewaltsame Dilatation hätte die dünne Wand zu sehr gefährdet, und wäre bei Fehlen des Sphincter zweeklos gewesen. Deshalb wurden Suppositoria mit Morphinm und Plumbum aceticum, Aloëpillen und Oelclysmata verordnet. Bei dieser. Behandlung heilten die Fissuren binnen kurzer Zeit, und bald danach trat wieder Conception ein, Die Schmerzen beim Coitus waren verschwunden.

Aehnliche Fälle sind wohl deshalb selten, weil bei grossen, un. vereinigt gebliebenen Dammrissen der Dickdarm gewöhnlich in einen Reizzustand geräth, dessen Folge chronische Diarrhoen sind.

Selbstrerständlich ist vor Allem die Perinaeoplastik indicirt.

1) Dentsches Archiv für klin. Med. XIII. 